\title{
Nociception Level (NoL) index alteration after standardized nociceptive stimulus decreases with higher doses of remifentanil
}

\author{
Marco Julien, Elizabeth Décary, Rami Issa, Olivier Verdonck, \\ Louis-Philippe Fortier, Philippe Richebé \\ Maisonneuve-Rosemont Hospital, Montréal, Canada
}

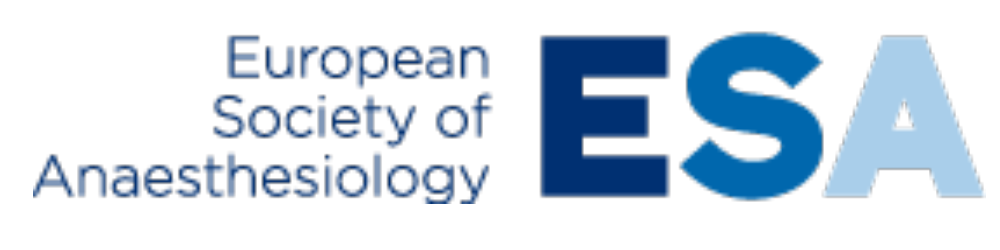

Background: Several indexes have been recently used to monitor nociception intensity during general anesthesia (GA) ${ }^{1}$. The PMD-100 monitor (Medasense Biometrics, Israel) is a novel monitor of nociception, presenting the Nociception Level (NoL) Index. The NoL index is a multiparametric index derived from heart rate $(\mathrm{HR}), \mathrm{HR}$ variability, plethysmograph wave amplitude, skin conductance and its fluctuations. This index ranges from 0 to 100 , with lower value meaning lower pain intensity ${ }^{2}$. We tested the NoL alteration during a standardized noxious stimulus at various doses of remifentanil (RF) i.v. infusion, with the hypothesis that the higher the RF dose, the lower the NoL alteration.

Methods: 40 patients received desflurane-RF based GA with an epidural analgesia for laparotomy. A moderate noxious stimulus (electrical stimulation $70 \mathrm{~mA}, 100 \mathrm{~Hz}, 30 \mathrm{sec}$ ) was applied to the forearm of the patients at 4 RF doses varying from 0.005 to $0.15 \mu \mathrm{g} \bullet \mathrm{kg}^{-1} \bullet \mathrm{min}^{-1}$. For each RF dosage, the pre- and the post-stimulation NoL peak values, the difference $(\triangle \mathrm{NoL})$ and $\mathrm{HR}$ were recorded and compared using a linear mixed effects model and Cl of 95\%. Study \# NCT02602379.

Results: The median pre stimulation NoL basal values ranged for 3 to 5 with no significant difference when RF infusion increased. The median post stimulation values at RF doses of $0.005,0.05,0.1$ and $0.15 \mu \mathrm{g} \bullet \mathrm{kg}^{-1} \bullet \mathrm{min}^{-1}$ were, respectively, $39,15,8$ and 8.5 , showing statistical significant difference between 0.005 and $0.05 \mu \mathrm{g} \bullet \mathrm{kg}^{-1} \bullet \mathrm{min}^{-1}(p<0.0001)$ and between 0.05 and $0.1 \mu \mathrm{g} \bullet \mathrm{kg}^{-1} \bullet \mathrm{min}^{-1}(p<0.01)$ but not at higher doses e.g. between 0.1 and $0.15 \mu \mathrm{g} \bullet \mathrm{kg}^{-1} \bullet \mathrm{min}^{-1}$ $(p=0.49)$. Accordingly, the $\Delta \mathrm{NoL}$ was greater at 0.005 than at $0.05 \mu \mathrm{g} \cdot \mathrm{kg}^{-1} \bullet \mathrm{min}^{-1}(26$ vs $6.5, \mathrm{p}<0.0001)$ and at 0.05 than at $0.1 \mu \mathrm{g} \bullet \mathrm{kg}^{-1} \bullet \mathrm{min}^{-1}(6.5$ vs $3, \mathrm{p}<0.01)$ but showed no difference between 0.1 and $0.15 \mu \mathrm{g}^{\circ} \mathrm{kg}^{-1} \bullet \mathrm{min}^{-1}$ (3 vs 3.5, $p=0.70)$. The correlation between RF dosage and poststimulation NoL value was higher $(\rho=0.61)$ than with $H R$ $(\rho=0.40)$

Conclusion: When a patient is exposed to a standardized noxious stimulation, NoL reaches higher peak values and shows greater alteration when the patient is receiving lower doses of RF. In this study, a plateau of minimal NoL variation was reached at $0.1 \mu \mathrm{g} \bullet \mathrm{kg}^{-1} \bullet \mathrm{min}^{-1}$ of $\mathrm{RF}$. The NoL index showed greater correlation with RF dose than HR did. These results suggest great potential of the NoL index as a tool to monitor nociception intensity during anesthesia. However, further studies are needed to assess whether the NoL index can better guide intraoperative opioid administration and monitor the nociception level than classic clinical signs like HR or blood pressure.
Figure 1. NoL pre and post stimulation value at different remifentanil dosage

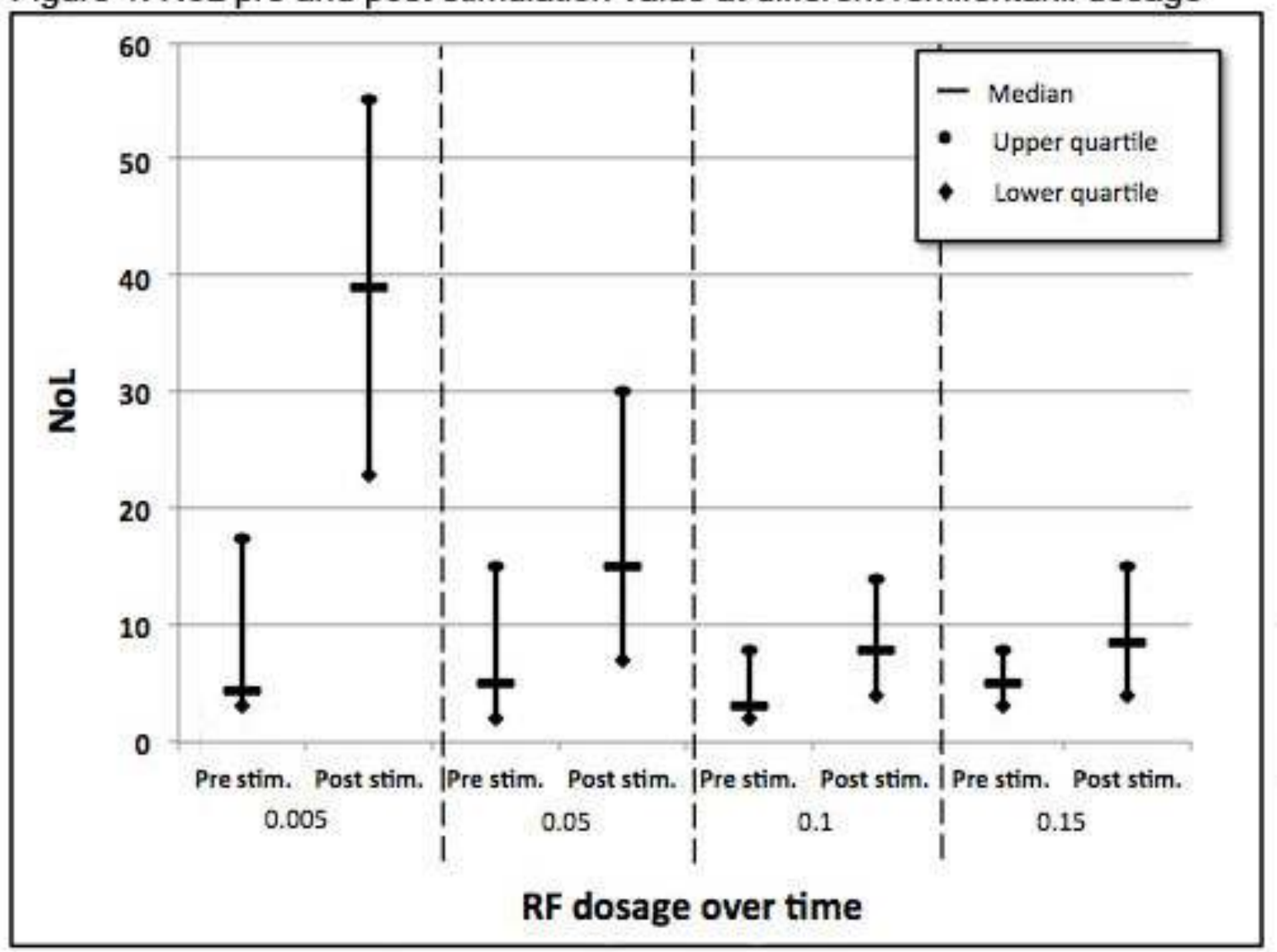

Comparison between pre and post stimulations at each time point was significant $(p<0.0001)$. A trend was observed in the pre stimulation RF dosage over time but was not significant $(p=0.0701)$.
The variation of NoL in the post stimulation RF dosage over time was significant $(p<0.0001)$. The correlation between RF dosage (post stim.) and Nol was significant $(p=-0.61, p<0.0001$ ).

Figure 2. Heart rate pre and post stimulation value at different remifentanil dosage

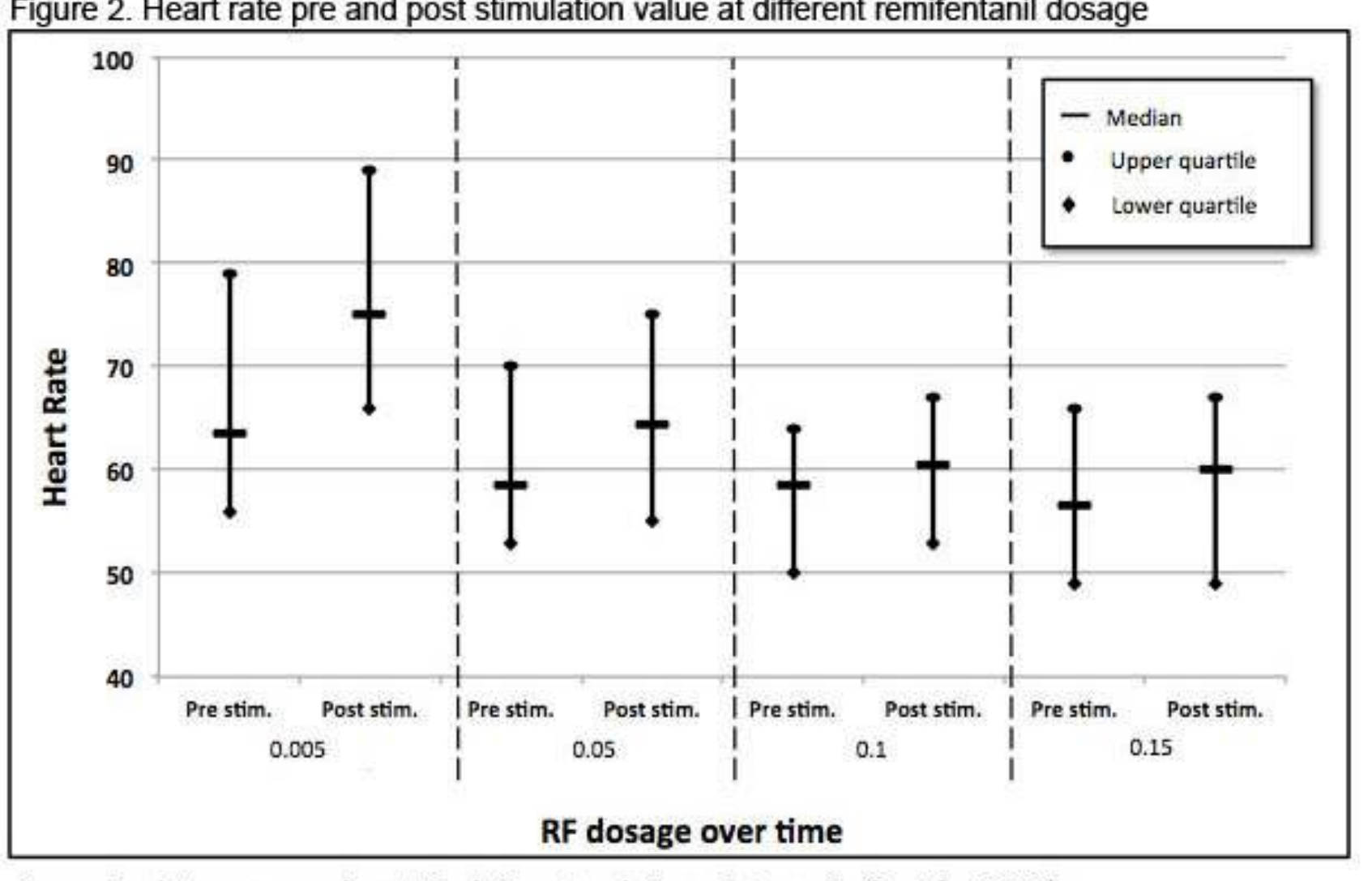

Comparison between pre and post stimulations at each time point was significant $(p<0.0001)$. The variation of heart rate in the pre stimulation RF dosage over time was significant ( $p=0.0007)$. The variation of heart rate in the post stimulation $\mathrm{RF}$ dosage over time was significant $(p<0.0001)$. The correlation between RF dosage (post stim.) and heart rate was significant $(\rho=-0.40, p<0.0001)$.

Figure 3. Pre and post stimulation difference in NoL value at different remifentanil dosage

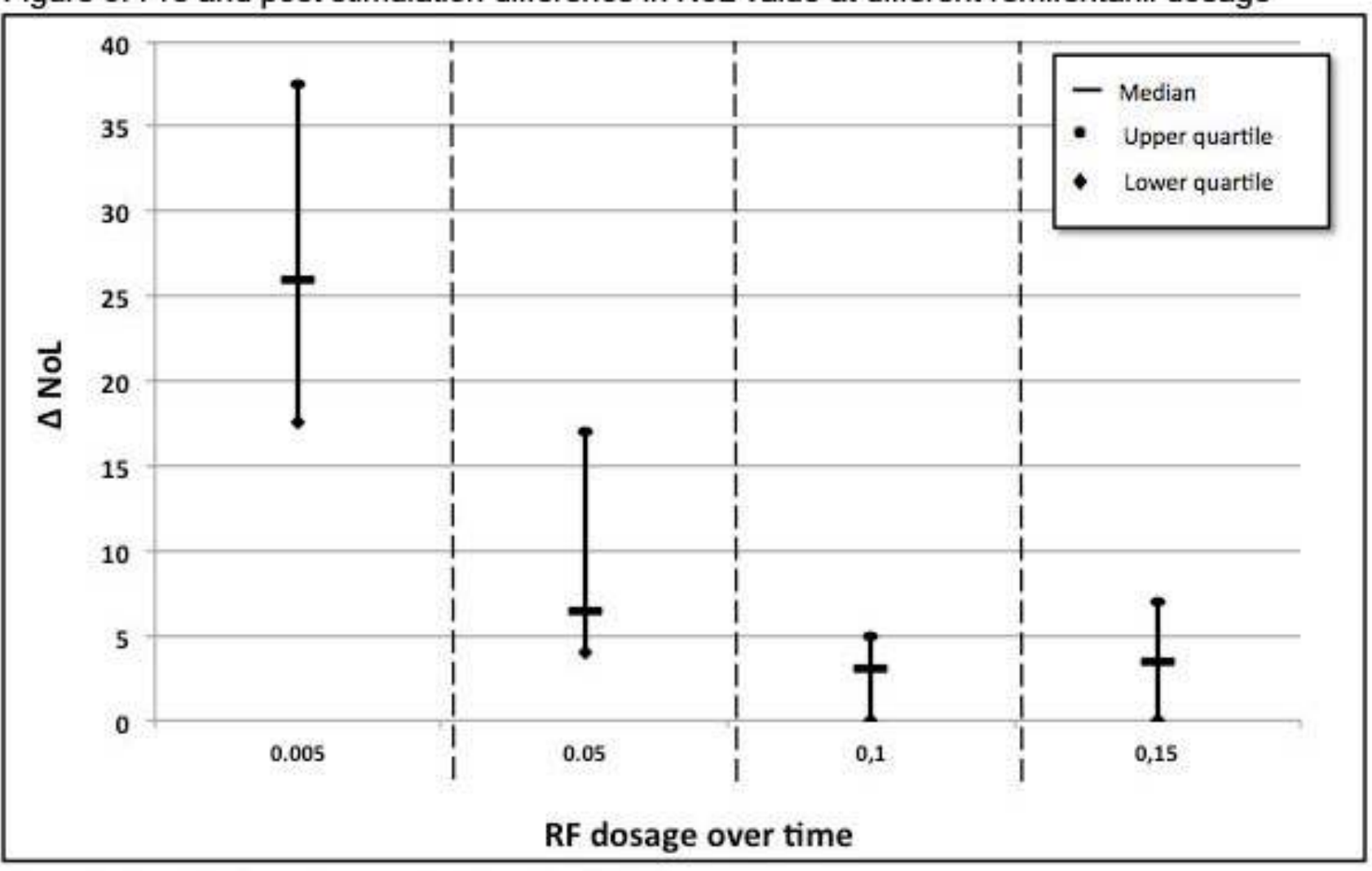

The variation of Nol over time was significant $(p<0.0001)$

The correlation between RF dosage and $\Delta \mathrm{Nol}$ was significant $(\rho=-0.61, p<0.0001)$.

1 Gruenewald M. et al. "Monitoring the nociception - anti-nociception balance" Best practice \& research clinical anaesthesiology 27 (2013) 235-247

2 Ben-Israel N. et al. "Monitoring the nociception level: a multi-parameter approch" J Clin Monit Comput 27 (2013) 659-668

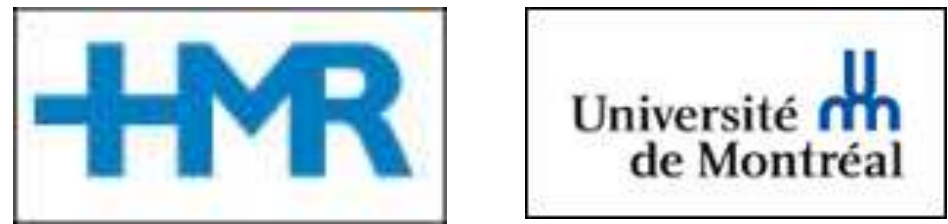

